\title{
Rapidly controlled outbreak of Serratia marcescens infection/colonisations in a neonatal intensive care unit, Pescara General Hospital, Pescara, Italy, April 2011
}

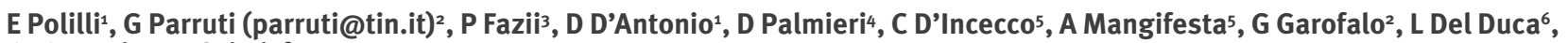

C D’Amario ${ }^{7}$ M Scimia ${ }^{8}$

1. Microbiology and Virology Unit, Santo Spirito General Hospital, Pescara, Italy

2. Infection Control Unit, Santo Spirito General Hospital, Pescara, Italy

3. Clinical Pathology Unit, Santo Spirito General Hospital, Pescara, Italy

4. Epidemiological Office, Santo Spirito General Hospital, Pescara, Italy

5. Neonatal Intensive Care Unit, Santo Spirito General Hospital, Pescara, Italy

6. Health Department Unit, Santo Spirito Pescara General Hospital, Pescara, Italy

7. General Direction, Pescara Health District, Pescara, Italy

8. Risk Management Unit, Pescara Health District, Pescara, Italy

Citation style for this article:

Polilli E, Parruti G, Fazii P, D’Antonio D, Palmieri D, D’Incecco C, Mangifesta A, Garofalo G, Del Duca L, D’Amario C, Scimia M. Rapidly controlled outbreak of Serratia marcescens infection/colonisations in a neonatal intensive care unit, Pescara General Hospital, Pescara, Italy, April $2011 .$.

Euro Surveill. 2011;16(24):pii=19892. Available online: http://www.eurosurveillance.org/ViewArticle.aspx?Articleld=19892

Article published on 16 June 2011

In April 2011, an outbreak of Serratia marcescens infection/colonisations occurred in the neonatal intensive care unit of Pescara General Hospital. Rapid microbiological investigations lead to identification of five cases of likely cross-transmission from a neonate hospitalised for $S$. marcescens sepsis: four infections and one neonate colonised post-mortem. Two low birth weight neonates died. The environmental investigation detected $S$. marcescens from two soap dispensers. Strict hygiene measures lead to early interruption of the outbreak, without recurrences to date.

Serratia marcescens is an opportunistic pathogen able to rapidly spread in the nosocomial environment, being identified in up to $16 \%$ of nosocomial Gram-negative bloodstream infections [1]. Several outbreaks of $S$. marcescens in neonatal intensive care unit (NICU) were documented in recent years [2-6], causing potentially fatal sepsis, meningitis or pneumonitis in very premature or low birth weight neonates with mortality rates as high as $44 \%$, significantly higher compared with those caused by Enterobacter cloacae, another well-known cause of disease and death in premature neonates $[2,7]$.

\section{Outbreak description and control measures}

In early April 2011, a normal weight neonate was born at 41 weeks of gestational age and was transferred to NICU of Pescara General Hospital because of fever, failure to thrive and increasing C-reactive protein (CRP) values. Blood cultures grew S. marcescens fully susceptible to quinolones and carbapenems. The neonate was treated with antibiotics for 11 days and discharged after full recovery on day 11. Three days after, four of 16 neonates in the NICU developed a clinical picture suggestive of sepsis, with rising
CRP values. All four were cared for in the same room. Aware of the recent case of $S$. marcescens, clinicians on duty asked for immediate testing and molecular characterisation by Septifast ${ }^{\circledR}$ (Roche) on blood samples which detected S. marcescens DNA from all four cases. Antibiotics were administered, based on susceptibility data from the possible source. Two of the four neonates died, after 20 and 22 days of hospitalisation. Both had been premature births (week 26 and 29) with birth weight $<1,200 \mathrm{~g}$. Due to respiratory distress at birth, they had been transferred to NICU directly from delivery rooms, 10 and four days before becoming symptomatic. The other two septic neonates were born at 31 and 38 weeks of gestational age, one with low and one with normal birth weight. They had been delivered through Caesarian section and transferred to NICU for respiratory distress, nine and five days before becoming septic. They were discharged in good condition after 49 and 25 days of hospitalisation respectively, after their CRP values were normal.

A fifth very low birth weight ( $1800 \mathrm{~g}$ ) neonate, cared for in the same room, had died the day before the four neonates became septic. Post-mortem sampling from his umbilical cord catheter grew S. marcescens, suggestive of colonisation.

\section{Environmental sampling}

On the day after the four neonates became symptomatic, extensive environmental microbiological investigations were started. Swabs were taken from numerous surfaces, including walls, floors with their edges and corners, doors and door handles, shelves, benches, hoods, sinks, cradles and ventilators, stethoscopes and other personal medical devices, milk 
collecting devices, medical records and trolleys. To address the possibility of human carriage, hands and cellular phones of some of the healthcare workers and auxiliary staff who worked in the particular room and of additional staff operating in other rooms in the NICU were checked. Bottles of saline used for dilutions and one phosphate buffer, drug bottles and boxes, soaps and disinfectants were either swabbed or cultured. Only two soap dispensers among all investigated environmental and human surfaces yielded $S$. marcescens isolates.

\section{Control measures}

The four infected neonates were cohorted in a single room and new admissions discontinued for 10 days.

Moreover, immediately after the microbiological sampling, a thorough sanitation procedure was started: possible sources were disposed and renewed, including parenteral nutrition, milk collection devices, disinfectants, soaps and soap dispensers, cotton and tissues. Already recommended measures, such as hand washing with alcohol based solutions at all sites in the ward, and using gloves when assisting and caring for neonates, whenever possible, were reinforced. Water and air filters had been routinely renewed a few days in advance and were left in place after negative cultures. After the first sampling, additional swabs were taken from 70 soap dispensers and from bottles of chlorhexidine-based hand disinfectants in stock. Microbiological sampling was extended to other potential sites of environmental persistence, including wards in the obstetrics and gynecology unit, delivery rooms and theatres for Caesarean sections. In these environments, floors, tissues, cradles, door handles and cabinets, sinks, soaps, soap dispensers, several surfaces in the delivery rooms, baby changing tables, scales, air ducts and filters in the nursery, all yielded negative results.

Repeated microbiological controls as of 14 June 2011 failed to identify any further environmental contamination with S. marcescens.

\section{Discussion}

The present outbreak involved six neonates. Five cases were due to likely cross-transmission from a neonate hospitalised for S. marcescens sepsis, four of whom were infected and one colonised post-mortem. Two neonates with clinical signs of sepsis were successfully treated with antibiotics. Two died likely from S. marcescens sepsis, leading to a mortality rate of $40 \%$. It is of note, that both deceased neonates had a positive epicutaneus culture for Klebsiella spp. after the isolation of S. marcescens, one of them having also a minor amplicon for K. pneumoniae at his molecular test. Both of them were premature, low weight $(\leq 1,200 \mathrm{~g}$ at birth) neonates.

The S. marcescens infections occurred at the same time, pointing towards a common exposure to a source(s) present in a narrow period of time in the NICU, after 18 years of negative environmental sampling.

Hand carriage, although not demonstrated in this outbreak, was the most likely way of spread to and from soap dispensers, of which two tested positive. This is in-line with the literature that shows that the organism can survive on the human skin for extended periods of time, and hand carriage is thought to be a mode of transmission when no environmental source is identified [3]. Moreover, other reports document soaps and detergents as potential site for the spread of $S$. marcescens [4].

In outbreaks with S. marcescens, extensive environmental microbiological investigations are needed to identify sources and reservoirs [2]. Environmental sources of S. marcescens in NICU include breast pumps, breast milk, parenteral nutrition, soap and disinfectant dispensers, laryngoscope blades, ventilators and air conditioning ducts $[2,6]$. Secondary and even tertiary waves of transmission have been recently reported [8-11]. Under such circumstances, strict measures such as nurse cohorting, closure of wards to new admissions and even temporary total closures of NICU were necessary to contain the spread of the microorganism $[10,11]$. After the implementation of strict sanitation and control measures in our NICU, there was no evidence of further transmission or environmental persistence of S. marcescens.

For this reason, molecular cluster analysis of the eight available isolates (six from the neonates and two from the soap dispensers) was not requested. Fortunately, this outbreak was limited to a small number of cases, with a single epidemic peak of colonisation/ infection and so far no recurrence. In recent NICU reports, greater numbers of patients were involved, the fraction of infected/colonised neonates among all neonates present in the wards higher, and environmental colonisation more protracted, with more than one epidemic peak; mortality rates were however comparable.

Prompt clinical suspicion of $S$. marcescens and the immediate use of a molecular assay allowed for early etiological diagnosis, supported by an epidemiological link with a recent case and paved the way to immediate measures for containment of nosocomial infections. These measures adopted before any evidence from traditional microbiological cultures, enabled the rapid interruption of the outbreak. Moreover, this report serves as a reminder of the importance to keep up hygiene precautions at any time specifically in high risk settings such as a NICU.

*Erratum: At the moment of publication, the names of $\mathrm{V}$ Cortesi and $\checkmark$ Fortunato were left out in the list of authors. This mistake was corrected on 21 June 2011 . We apologise to the authors. 


\section{References}

1. Graham PL 3rd, Begg MD, Larson E, Della-Latta P, Allen A, Saiman L. Risk factors for late onset gram-negative sepsis in low birth weight infants hospitalized in the neonatal intensive care unit. Pediatr Infect Dis J. 2006;25(2):113-7.

2. Voelz A, Muller A, Gillen J, Le C, Dresbach T, Engelhart S, et al. Outbreaks of Serratia marcescens in neonatal and pediatric intensive care units: clinical aspects, risk factors and management. Int J Hyg Environ Health. 2010;213(2):79-87.

3. Macdonald TM, Langley JM, Mailman T, Allain K, Nelson G, Hatton L, et al. Serratia marcescens outbreak in a neonatal intensive care unit related to the exit port of an oscillator. Pediatr Crit Care Med. 2011. Forthcoming.

4. Buffet-Bataillon S, Rabier V, Betremieux P, Beuchee A, Bauer $M$, Pladys $P$, et al. Outbreak of Serratia marcescens in a neonatal intensive care unit: contaminated unmedicated liquid soap and risk factors. J Hosp Infect. 2009;72(1):17-22.

5. Arslan U, Erayman I, Kirdar S, Yuksekkaya S, Cimen O, Tuncer I, et al. Serratia marcescens sepsis outbreak in a neonatal intensive care unit. Pediatr Int. 2010;52(2):208-12.

6. Rabier V, Bataillon S, Jolivet-Gougeon A, Chapplain JM Beuchee A, Betremieux P. Hand washing soap as a source of neonatal Serratia marcescens outbreak. Acta Paediatr. 2008;97(10):1381-5

7. Anderson B, Nicholas S, Sprague B, Campos J, Short B, Singh N. Molecular and descriptive epidemiologyof multidrug. resistant Enterobacteriaceae in hospitalized infants. Infect Control Hosp Epidemiol. 2008;29(3):250-5.

8. Crivaro V, Bagattini M, Salza MF, Raimondi F, Rossano F, Triass $M$, et al. Risk factors for extended-spectrum beta-lactamaseproducing Serratia marcescens and Klebsiella pneumoniae acquisition in a neonatal intensive care unit. J Hosp Infect. 2007;67(2):135-41.

9. Casolari C, Pecorari M, Fabio G, Cattani S, Venturelli C, Piccinini L, et al. A simultaneous outbreak of Serratia marcescens and Klebsiella pneumoniae in a neonatal intensive care unit. J Hosp Infect. 2005;61(4):312-20.

10. Villari P, Crispino M, Salvadori A, Scarcella A. Molecular epidemiology of an outbreak of Serratia marcescens in a neonatal intensive care unit. Infect Control Hosp Epidemiol. 2001;22(10):630-4.

11. Fleisch F, Zimmermann-Baer U, Zbinden R, Bischoff G, Arlettaz $R$, Waldvogel K, et al. Three consecutive outbreaks of Serratia marcescens in a neonatal intensive care unit. Clin Infect Dis. 2002;34(6):767-73. 